Joint ACE Ground Penetrating Radar Antenna Test Facility at the Technical University of Denmark

H.-R. Lenler-Eriksen ${ }^{1}$, P. Meincke*1, A. Sarri ${ }^{2}$, V. Chatelée ${ }^{3}$, B. Nair ${ }^{4}$, I. J. Craddock ${ }^{5}$, G. Alli ${ }^{2}$, J.-Y. Dauvignac ${ }^{3}$, Y. Huang ${ }^{4}$, D. Lymperopoulos $^{6}$, and R. Nilavalan ${ }^{5}$

${ }^{1} \emptyset$ rsted.DTU, Build. 348, Technical University of Denmark, DK-2800

Kgs. Lyngby, Denmark

${ }^{2}$ IDS - Ingegneria Dei Sistemi S.p.A., Via Livornese 1019, 56010 Pisa, Italy

${ }^{3}$ Centre National de la Recherche Scientifique, Laboratoire

d'Electronique, Antennes et Télécommunications (CNRS/LEAT), 250 rue Albert Einstein, 06560 Valbonne, France

${ }^{4}$ Dept. of Electrical Engineering and Electronics, University of Liverpool, Brownlow Hill, Liverpool L69 3GJ, United Kingdom

${ }^{5}$ Dept. of Electrical and Electronic Engineering, Merchant Venturers Building, Woodland Road, Bristol BS8 1UB, United Kingdom

${ }^{6}$ Microwave and Fiber Optics Laboratory, Institute of Communications and Computer Systems, School of Electrical and Computer Engineering, Iroon Polytexneiou St. Zografou, Athens Attiki, GR 15780, Greece

A Ground Penetrating Radar (GPR) antenna test facility, established within the ACE network at the Technical University of Denmark (DTU), is described. Examples of results from the facility obtained from measurements of eight different GPR antennas are presented.

\title{
Description of Facility
}

The ACE GPR antenna test facility, shown in Figure 1, has been established in order to enable comparisons of GPR antenna radiation characteristics in a controlled and consistent environment. The facility consists of a soil-filled wooden box with length $3.8 \mathrm{~m}$, width $3.3 \mathrm{~m}$, and depth $1 \mathrm{~m}$, see Figure 2. Above the box a mechanicalscanning system is installed, able to move the GPR Antenna Under Test (AUT) along two orthogonal directions in a plane parallel to the air-soil interface. The scanning of the AUT is performed by two motors connected to associated motor controllers.

Figure 3 shows the mounting arrangement for the AUT. The cylindrical rod, to which the AUT is attached, has a diameter of $22 \mathrm{~mm}$. The rod has a number of holes for a pawl of diameter $6 \mathrm{~mm}$. The pawl is used to set the distance from the AUT to the air-soil interface, as well as to rotate the AUT to control the measurement of two orthogonal polarizations.

At the center of the box a probe is buried at a depth of $20.3 \mathrm{~cm}$. The probe is a loop antenna with radius $17.8 \mathrm{~mm}$, made of semi-rigid cable UT-141A with wire diameter $3.5 \mathrm{~mm}$ and total length $5 \mathrm{~m} \mathrm{[1]}$. Figure 4 shows the probe mounted on a supporting plastic rod. The semi-rigid cable is connected to one port of a HP8753A 


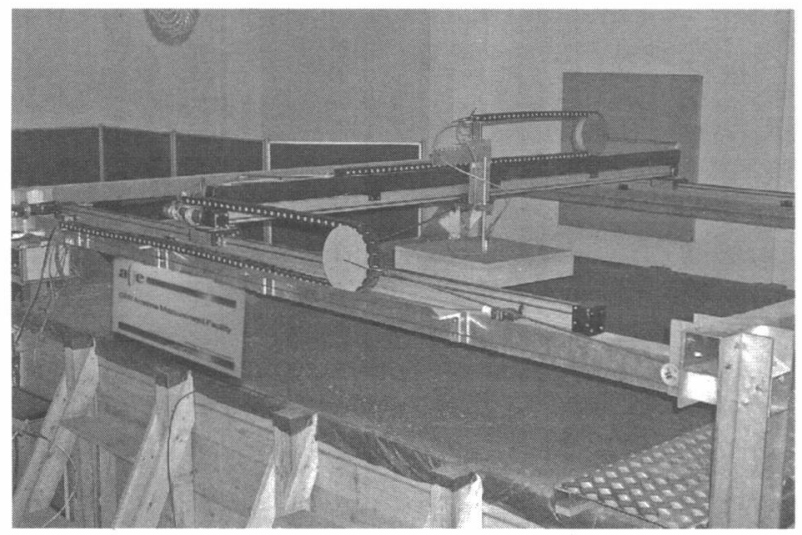

Figure 1: The GPR facility at DTU.
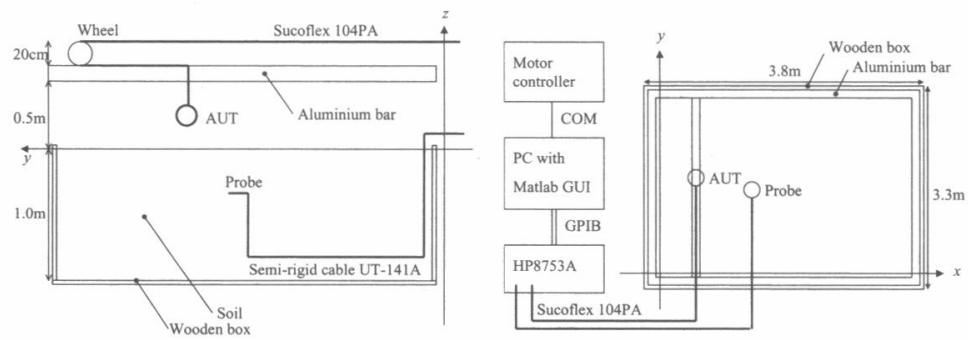

Figure 2: The setup of the facility.

vector network analyzer, and the other port is attached to the AUT through a phasestable Sucoflex 104PA coaxial cable. The HP8753A and the motors of the scanning system are controlled by a PC with a MATLAB GUI, as shown in Figure 2.

For each desired position of the AUT, the network analyzer measures the scattering matrix parameter $S_{12}=V_{1}^{-} /\left.V_{2}^{+}\right|_{V_{1}^{+}=0}$. Herein, $V_{1}^{+}$and $V_{1}^{-}$denote the voltages of the incident and reflected waves, respectively, in the coaxial cable attached to the AUT, and similar definitions hold for $V_{2}$ for the probe. From the measured scattering matrix parameter $S_{12}$ various information about the AUT can be extracted.

\section{Example of Measurement Results}

Eight GPR antennas from the ACE community were measured at the facility during one week in December 2004, the intention being to compare these antennas under consistent ground conditions. The antennas were three Exponentially-Tapered Slot Antennas (ETSAs) from CNRS/LEAT, two loaded bow-tie shaped antennas from 


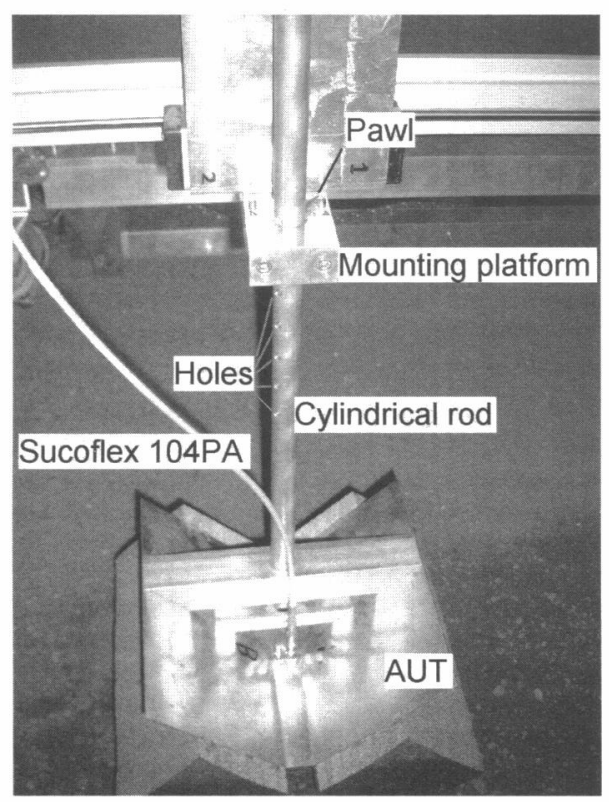

Figure 3: The mounting platform of the AUT.

IDS in Italy, two spiral antennas from University of Liverpool, and a patch antenna from Institute of Communications and Computer Systems in Athens. Figure 5 shows one of the measured antennas, IDS TR1600, mounted on the AUT platform. The footprints at $1.14 \mathrm{GHz}$ of the linear co- and cross-polarized components of the AUT are seen in Figure 6. The footprints are based on the above-mentioned measured scattering matrix parameters with the reference plane moved from the end of the semi-rigid cable to the excitation cut of the loop antenna. No probe correction is performed, that is, the finite extent of the probe is not accounted for. More advanced processing, including probe correction, can be carried out on the basis of the procedures in [2]. Due to space constraints, results for the other antennas are not provided here but will be available for the conference.

\section{References}

[1] A. G. Yarovoy, R. V. D. Jongh, and L. P. Ligthart, "Ultra-wideband sensor for electromagnetic field measurements in time domain," IEE Electronics Letters, pp. 1679-1680, Sept. 2000.

[2] P. Meincke and T. B. Hansen, "Plane-wave characterization of antennas close to a planar interface," IEEE Trans. Geosci. Remote Sensing, pp. 1222-1232, June 2004. 


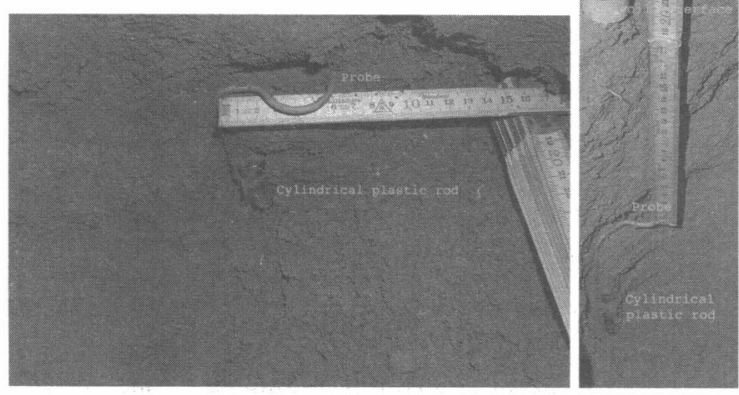

Figure 4: The probe mounted on the plastic rod in the soil.

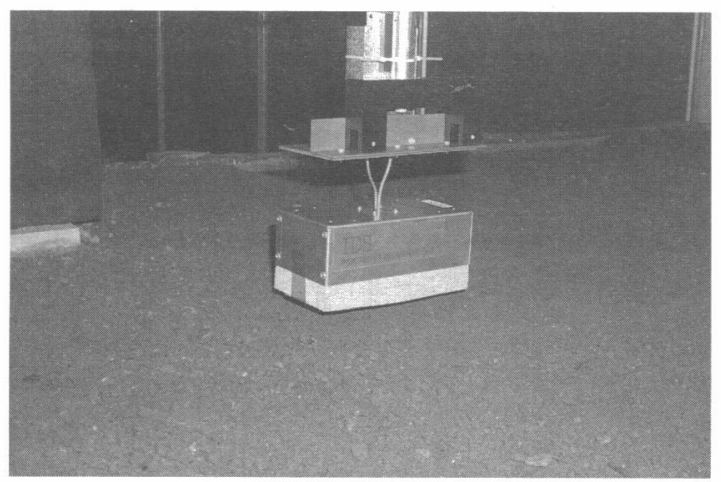

Figure 5: IDS TR1600.
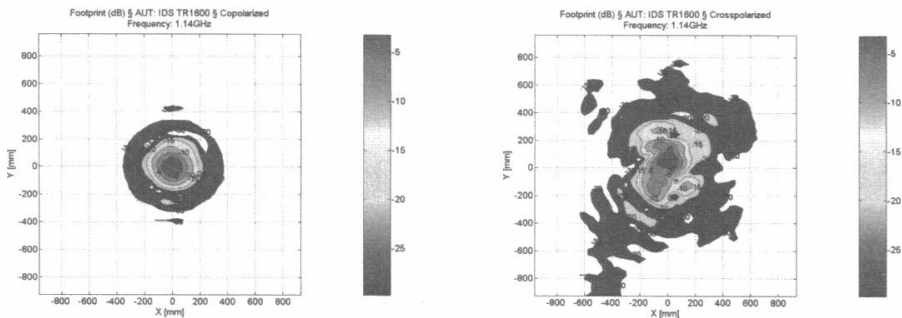

Figure 6: Footprints for the co- and cross-polarized components of the AUT. The data in each plot are normalized with respect to the maximum and the difference between the maxima of the co- and cross-polarized components is $13.8 \mathrm{~dB}$. 